\title{
Stochastic Analysis of a Single-Degree-of-Freedom Nonlinear Experimental Moored System Using an Independent-Flow-Field Model
}

\author{
H. Lin, A.M.ASCE, ${ }^{1}$ and S. C. S. Yim, M.ASCE ${ }^{2}$
}

\begin{abstract}
Stochastic characteristics of the surge response of a nonlinear single-degree-of-freedom moored structure subjected to random wave excitations are examined in this paper. Sources of nonlinearity of the system include a complex geometric configuration and wave-induced quadratic drag. A Morison-type model with an independent-flow-field formulation and a three-term-polynomial approximation of the nonlinear restoring force is employed for its proven excellent prediction capability for the experimental results investigated. Wave excitations considered in this study include nearly periodic waves, which take into account the presence of tank noise, noisy periodic waves that have predominant periodic components with designed additive random perturbations, and narrow-band random waves. A unified wave excitation model is used to describe all the wave conditions. A modulating factor governing the degree of randomness in the wave excitations is introduced. The corresponding Fokker-Planck formulation is applied and numerically solved for the response probability density functions (PDFs). Experimental results and simulations are compared in detail via the PDFs in phase space. The PDFs portray coexisting multiple response attractors and indicate their relative strengths, and experimental response behaviors, including transitions and interactions, are accordingly interpreted from the ensemble perspective. Using time-averaged probability density functions as an invariant measure, probability distributions of large excursions in experimental and simulated responses to various random wave excitations are demonstrated and compared. Asymptotic long-term behaviors of the experimental responses are then inferred.
\end{abstract}

DOI: 10.1061/(ASCE)0733-9399(2004)130:2(1)

CE Database subject headings: Experimental data; Nonlinear systems; Structural analysis; Probabilistic methods; Probability density functions; Mooring; Wave forces; Stochastic processes.

\section{Introduction}

Complex nonlinear response phenomena of compliant ocean structural systems subjected to periodic wave excitations, including nonharmonics, resonances, and coexistence have been studied analytically and demonstrated via computer simulations (Virgin and Bishop 1988; Thompson 1989; Soliman and Thompson 1991; Virgin and Erickson 1994; Gottlieb and Yim 1992, 1993). Nonlinearities of this class of systems are usually induced by a combination of geometrically nonlinear restoring force and (quadratic) drag force. In a deterministic setting, Gottlieb and Yim (1992, 1993) conducted local and global stability analyses of moored ocean structures modeled as simple single-degree-offreedom (SDOF) systems, and estimated stability regions of various nonlinear responses. Using the simple models, Gottlieb et al. (1997) identified the existence of underlying organized transitions

\footnotetext{
${ }^{1}$ Research Associate, Civil, Construction and Environmental Engineering Dept., Oregon State Univ., Corvallis, OR 97331.

${ }^{2}$ Professor,Civil, Construction and Environmental Engineering Dept., Oregon State Univ., Corvallis, OR 97331.

Note. Associate Editor: Roger G. Ghanem. Discussion open until July 1, 2004. Separate discussions must be submitted for individual papers. To extend the closing date by one month, a written request must be filed with the ASCE Managing Editor. The manuscript for this paper was submitted for review and possible publication on September 17, 2002; approved on July 8, 2003. This paper is part of the Journal of Engineering Mechanics, Vol. 130, No. 2, February 1, 2004. CASCE, ISSN 0733-9399/2004/2$1-9 / \$ 18.00$.
}

embedded in bifurcation domains and developed analytical procedures to facilitate a numerical search of possible routes to chaos.

In addition to the analytical and numerical investigations, Yim et al. (1993) also conducted experimental studies to verify the analytical predictions in deterministic settings. Nonlinear responses including subharmonics and superharmonics and bifurcations were observed experimentally. Underlying bifurcation superstructures were detected, and the existence of higher order nonlinear responses (e.g., quasiperiodic and chaotic) was indicated (Lin et al. 1998). They observed some "unexpected" transitions (e.g., harmonic transition to subharmonic) in experimental response characteristics in the designed "deterministic" settings. For these cases, small variations in wave amplitude were noted.

A preliminary study of the experimental results was carried out by employing a simple, standard Morison (SM) type nonlinear model (Lin and Yim 1998). The geometric-nonlinear restoring force was approximated by two-term polynomials (linear and cubic) at static equilibrium. The wave-frequency dependency of the hydrodynamic properties was taken into account by identifying system parameters for each and every of the sample testsvarious (inconsistent) parameter sets were identified in different frequency ranges. Numerical predictions were in reasonably good agreement with experimental results. The model, however, failed to determine a set of constant coefficients to closely predict overall response behavior over the frequency range experimentally considered.

In identifying a model to improve prediction capability of the experimental results, an investigation of modeling and parameter 
identification of the experiment had been recently carried out by Narayanan and Yim (2000). Numerical results indicated that an independent-flow-field (IFF) Morison model with three-term polynomial approximation for the restoring force was the most suitable for the chosen experimental results. A set of "optimal" constant coefficients was later identified by Yim and Lin (2002) for the wave frequency range considered. With this set of constant coefficients, simulations of the IFF model were in good agreement with the experimental results.

Noting the noise effects on the structural responses (Yim et al. 1993), Yim and Lin carried out a two-part study as a first attempt to examine the experimental responses under noisy periodic wave excitations. They employed a standard Morison type of model with two-term polynomial approximation of the nonlinear restoring force and a systematic probabilistic analysis procedure, including the Fokker-Planck formulation with a path-integral solver. They laid out the framework of probabilistic analysis on the model in Part I (Lin and Yim 1997), and employed the resulting analytical predictions to quantitatively interpret the experimental results in Part II (Yim and Lin 2000). They found that the model could predict well response behaviors near the primary resonance, but could not capture the nonlinear behaviors, especially the sub- and superharmonics and coexistence of multiple attractors near the secondary resonances. In their study, examinations of the responses subjected to other measured wave excitations, e.g., nearly periodic and narrow-band, were not included. They suggested a more suitable model be developed and more comprehensive, detailed comparisons be conducted.

In this study, we systematically and consistently examine and analyze all the experimental results over the entire frequency range and noise levels from a stochastic perspective taking into account the random nature of the wave excitations in the experiment reported by Yim et al. (1993). Experimental wave excitations are accordingly classified as nearly periodic, noisy periodic, and narrow-band random waves. As an extension of the previous studies (Yim and Lin 2000), detailed comparisons of analytical predictions and experimental results are carried out in the probabilistic domain via response probability density functions (PDFs). An alternative Morison-type model incorporating an independentflow-field (IFF) formulation with a unified wave excitation presentation is employed. The IFF model consists of an alternative form of Morison hydrodynamic damping (independent-flow-field) and a three-term-polynomial (including an additional quadratic term) approximation to the nonlinear restoring force. The ranges of system parameters were first estimated using those provided by Narayanan and Yim (2000) employing a frequency-domain identification technique. An optimal set of consistent, constant parameters for the wave frequencies considered was later identified (Yim and Lin 2002) and is employed here. The previous studies show the model predictions are in very good agreement with experimental results in individual response characteristics and overall response behaviors. Wave excitations considered, including nearly periodic, noisy periodic, and narrow-band random waves are presented by a unified wave excitation model with a modulation factor governing the degree of randomness.

The stochastic analysis procedure includes the Fokker-Planck equation (FPE) and its path-integral solver (Naess and Johnsen 1991; Lin and Yim 1995, 1997). Response probability density functions (PDFs) are computed by solving the associated FPE, and then compared in detail with experimental results. Probabilistic characteristics and relative strengths of coexisting multiple response attractors are depicted and examined via the PDFs. Effects of various types of randomness, e.g., random perturbations a)
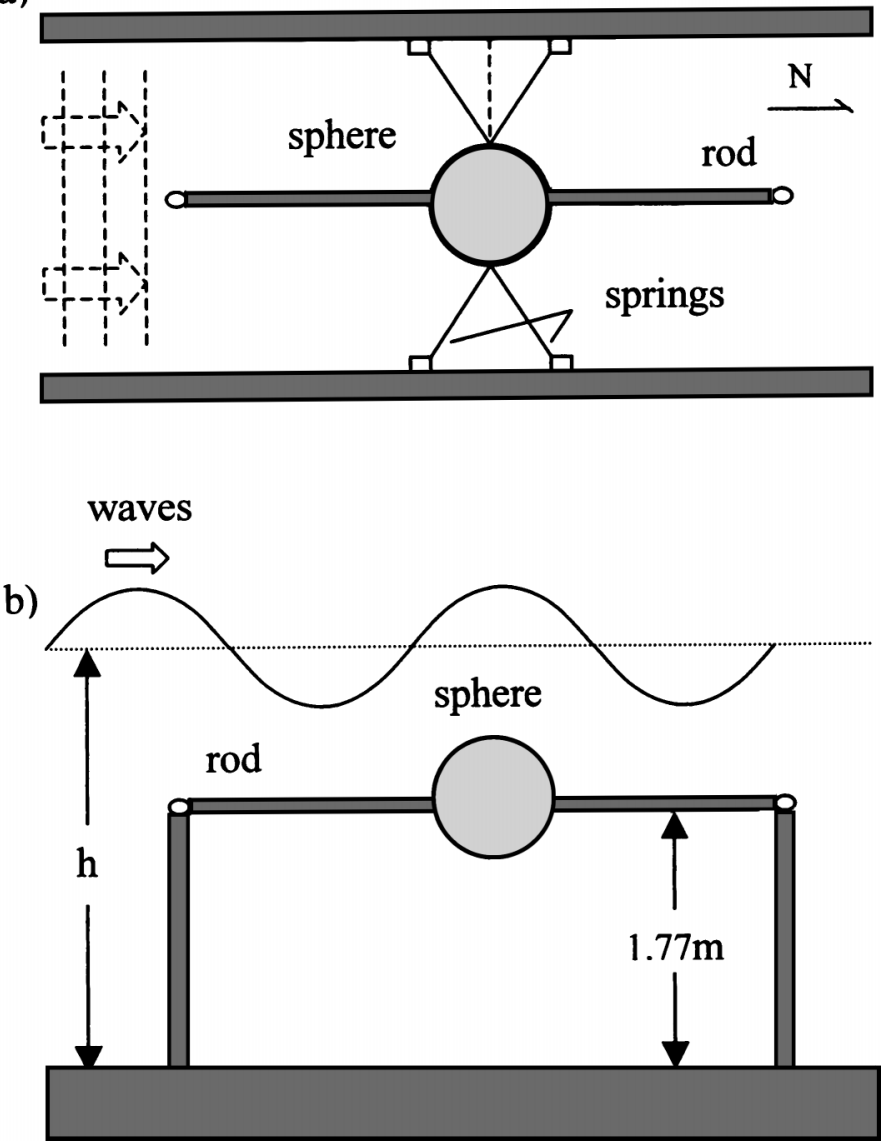

Fig. 1. Experimental model of a submerged, hydrodynamically damped and excited nonlinear structural system

and narrow band with varied intensities in the wave excitations, are examined and discussed. Time-averaged PDFs are computed and used as an invariant measure of nonlinear stochastic responses. Using the calculated approximate measures and Rice's formula, distributions of large excursions of the model responses are estimated (Naess and Johnsen 1993).

\section{System Description}

The multipoint moored structural system considered in this study is formulated as a SDOF submerged rigid body, hydrodynamically damped and excited nonlinear oscillator with random perturbations.

\section{Model Configuration}

The configuration of the experimental model consisted of a sphere on a rod, which was supported by guyed masts $6 \mathrm{ft}$ above the bottom of the wave flume (Fig. 1). The PVC sphere of $0.4572 \mathrm{~m}$ diameter was submerged and filled with water. The sphere weighed (in air) $11.35 \mathrm{~kg}$ empty and $48.12 \mathrm{~kg}$ filled. The rod restrained free-play of the sphere, thus the model behaved as a SDOF system in surge. Two Delrin bearings were placed on the sphere to minimize the friction between the sphere and the rod. The $90^{\circ}$ configuration (geometry parameter $\beta=0$ ), with the highest degree of nonlinearity in the restoring force, was chosen for its rich complex nonlinear phenomena. Two springs of stiffness 
291.86 N/m were then attached to the sphere at angles of $90^{\circ}$ to provide a nonlinear restoring force. Initial tension in each spring was set at $111.2 \mathrm{~N}$ to ensure the model response frequently reaching the nonlinear regime.

\section{Equations of Motion}

The response behavior of a hydrodynamically driven moored structure can be modeled by the following set of first-order nonlinear ordinary differential equations (Narayanan and Yim 2000):

$$
\begin{gathered}
\dot{x}_{1}=x_{2} \\
\dot{x}_{2}=\frac{1}{\left(M+M_{a}\right)}\left[-R^{\prime}\left(x_{1}\right)-C_{S}^{\prime} x_{2}-C_{D}^{\prime}\left|x_{2}\right| x_{2}+F_{D}^{\prime}+F_{I}^{\prime}\right]
\end{gathered}
$$

where $x_{1}$ and $x_{2}=$ surge displacement and velocity, respectively; $M=$ sphere mass; $M_{a}=$ the added mass; $C_{S}^{\prime}=$ structural damping coefficient; and $C_{D}^{\prime}=$ drag coefficient in an alternative Morisontype formulation [independent flow fields (IFF)]. $R^{\prime}=$ system restoring force (due to nonlinear geometric configuration), which is given by

$$
R^{\prime}=\alpha\left[x_{1}-\tau\left(\frac{l_{1}+l_{2}}{l_{1} l_{2}} x_{1}\right)\right] \cong K_{1} x_{1}+K_{2} x_{1}^{2}+K_{3} x_{1}^{3}
$$

where $l_{1,2}=$ time-varying lengths of the two pairs of the mooring lines, respectively; $\alpha=$ stiffness parameter; and $\tau=$ constant of initial tensions in the mooring lines. Good agreement between the taut mooring restoring force and the three-term polynomial approximation $(1 b)$ in the response range considered has been demonstrated by Narayanan and Yim (2000). Assume that the randomly perturbed wave profile $\eta(t)$ can be represented by the combination of a dominant periodic component and additive noise perturbation as given by

$$
\eta(t)=A \cos (\omega t+\phi)+\xi_{\eta}(t)
$$

where $A, \omega$, and $\phi=$ wave amplitude, frequency, and phase shift, respectively; and $\xi_{\eta}=$ additive noise. The drag force $F_{D}^{\prime}$ is then composed of deterministic component ${ }^{d} F_{D}^{\prime}$ and additive random component $\xi_{D}(t)$ as given by

$$
F_{D}^{\prime}=\frac{1}{2} \rho C_{D} A_{P}|u| u \approx \frac{1}{2} \rho C_{D} A_{P}\left|u^{d}\right| u^{d}+\xi_{D}(t)={ }^{d} F_{D}^{\prime}+\xi_{D}(t)
$$

where $\rho=$ water density; $C_{D}=$ drag coefficient; $A_{P}=$ projected area; and $u$ and $u^{d}=$ perturbed and deterministic fluid particle velocities, respectively. Similarly, $F_{I}^{\prime}$ represents the perturbed inertial force with combined deterministic component ${ }^{d} F_{I}^{\prime}$ and additive random component $\xi_{I}(t) . F_{I}^{\prime}$ is given by

$$
F_{I}^{\prime}=\rho C_{m} \forall \frac{\partial u}{\partial t} \approx \rho C_{m} \forall \frac{\partial u^{d}}{\partial t}+\xi_{I}(t)={ }^{d} F_{I}^{\prime}+\xi_{I}(t)
$$

with $C_{m}=$ inertial coefficient; and $\forall=$ sphere volume.

Stochastic wave excitations considered in the experiment, including nearly periodic, noisy periodic, and narrow-band random waves are approximated by a unified presentation. Eq. (1) can be rewritten as

$$
\begin{gathered}
\dot{x}_{1}=x_{2} \\
\dot{x}_{2}=-R\left(x_{1}\right)-C_{s} x_{2}-C_{d}^{\prime}\left|x_{2}\right| x_{2}+\sqrt{1-\gamma^{2}}\left({ }^{d} F_{D}+{ }^{d} F_{I}\right)+\gamma \xi(t)
\end{gathered}
$$

where $R\left(x_{1}\right)=R^{\prime} /\left(M+M_{a}\right)=k_{1} x_{1}+k_{2} x_{1}^{2}+k_{3} x_{1}^{3}, \quad C_{S}=C_{S}^{\prime} /(M$ $\left.+M_{a}\right), \quad C_{d}^{\prime}=C_{D}^{\prime} /\left(M+M_{a}\right), \quad{ }^{d} F_{D}={ }^{d} F_{D}^{\prime} /\left(M+M_{a}\right), \quad$ and ${ }^{d} F_{I}$
$={ }^{d} F_{I}^{\prime} /\left(M+M_{a}\right)$. Parameter $\gamma$ is the modulating factor governing the degree of randomness. When $\gamma=0$, the wave is deterministic and periodic, and when $\gamma=1$, the wave is random with narrow spectral bandwidth. $\xi(t)\left\{=\left[\xi_{D}(t)+\xi_{I}(t)\right] /\left(M+M_{a}\right)\right\}$ with variance $\sigma_{\xi}^{2}$, approximating all possible stochastic components in the wave excitations is a narrow-band process, which is obtained from a linearly filtered white noise. The linear filter is given by

$$
\ddot{\xi}(t)+\beta_{n} \dot{\xi}(t)+\left(2 \pi f_{0}\right)^{2} \xi(t)=\zeta(t)
$$

where $\zeta=$ delta-correlated white noise with unit intensity (Lin and Yim 1997). System parameters and hydrodynamic properties are estimated by a frequency domain identification techniquereverse multi-input-single-output (R-MISO) (Narayanan and Yim 2000). By comparing with experimental results in the time domain, an optimal set of constant parameters has been identified in good agreement with experimental responses over the frequency range considered (Yim and Lin 2002).

\section{Fokker-Planck Formulation}

The associated Fokker-Planck equation and its path-integral solver are derived here to solve for response PDFs for later data analysis and behavior predictions. The Fokker-Planck equation is given by

$$
\begin{aligned}
\frac{\partial P(X, t)}{\partial t}= & -\frac{\partial}{\partial x_{1}}\left[x_{2} P(X, t)\right]-\frac{\partial}{\partial x_{2}}\left\{\left[-R\left(x_{1}\right)-C_{s} x_{2}-C_{d}^{\prime}\left|x_{2}\right| x_{2}\right.\right. \\
& \left.\left.+\sqrt{1-\gamma^{2}} F+\gamma x_{3}\right] P(X, t)\right\}-\frac{\partial\left[x_{4} P(X, t)\right]}{\partial x_{3}} \\
& +\frac{\partial\left\{\left[-\beta_{n} x_{4}-\left(2 \pi f_{0}\right)^{2} x_{3}\right] P(X, t)\right\}}{\partial x_{4}}+\frac{\kappa}{2} \frac{\partial^{2} P(X, t)}{\partial x_{4}^{2}}
\end{aligned}
$$

where

$$
\begin{gathered}
x_{1}=x, \quad x_{2}=\dot{x}, \quad x_{3}=\xi, \quad x_{4}=\dot{\xi} \\
F={ }^{d} F_{D}+{ }^{d} F_{I} \\
P(X, t)=P\left(x_{1}, x_{2}, x_{3}, x_{4}, t\right)
\end{gathered}
$$

and the path-integral solution is given by

$$
\begin{aligned}
P(X, \mathrm{~d} t)= & \lim _{\substack{\mathrm{d} t \rightarrow 0 \\
N \rightarrow \infty \\
N \mathrm{~d} t \rightarrow t-t_{0}}} \prod_{i=0}^{N-1} \int \cdots \\
& \times \int \exp \left[-\mathrm{d} t \sum_{j=0}^{N-1} G\left(X^{j+1}, X^{j}, t^{j} ; \mathrm{d} t\right) P\left(X_{0}, t_{0}\right)\right] \mathrm{d} X_{i}
\end{aligned}
$$

with the short time propagator $G\left(X^{\prime}, X, t ; \mathrm{d} t\right)$ given as

$$
\begin{aligned}
& G\left(X^{j+1}, X^{j}, t^{j} ; \mathrm{d} t\right) \\
& =(2 \pi \mathrm{d} t)^{-4} \kappa^{-1 / 2} \exp \left[-\frac{\mathrm{d} t}{2 \kappa}\left(-\beta_{n} x_{4}^{j}-\left(2 \pi f_{0}\right)^{2} x_{3}^{j}\right.\right. \\
& \left.\left.\quad-\frac{x_{4}^{j+1}-x_{4}^{j}}{\mathrm{~d} t}\right)^{2}\right] \delta\left(-C_{s} x_{2}^{j}-C_{d}^{\prime} x_{2}^{j}\left|x_{2}^{j}\right|-R\left(x_{1}^{j}\right)\right.
\end{aligned}
$$


a)

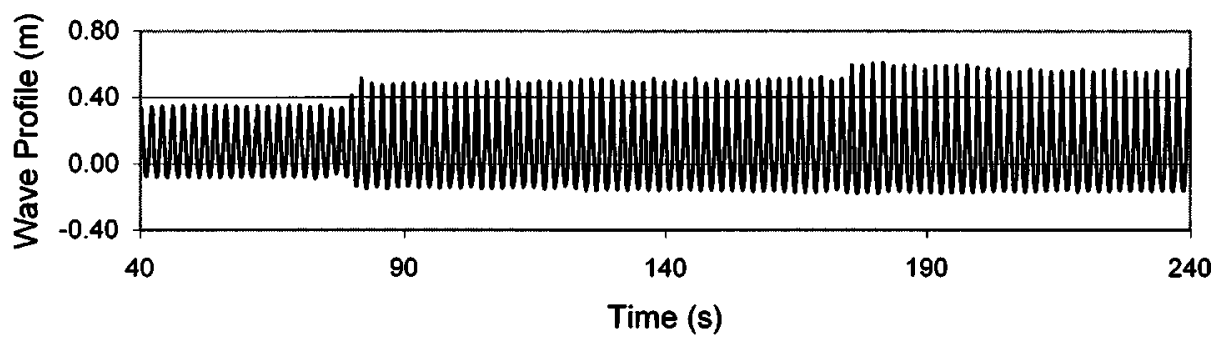

b)

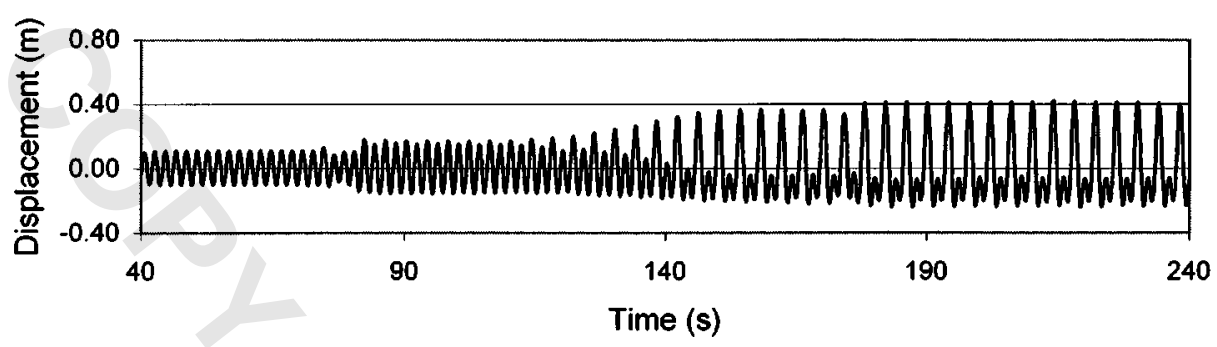

Fig. 2. Transition from harmonics to subharmonics (test D2): (a) wave profile and (b) sphere displacement

$$
\begin{aligned}
& \left.+\sqrt{1-\gamma^{2}} F^{j}+\gamma x_{3}^{j}-\frac{x_{2}^{j+1}-x_{2}^{j}}{\mathrm{~d} t}\right) \delta\left(x_{4}^{j}-\frac{x_{3}^{j+1}-x_{3}^{j}}{\mathrm{~d} t}\right) \\
& \times \delta\left(x_{2}^{j}-\frac{x_{1}^{j+1}-x_{1}^{j}}{\mathrm{~d} t}\right)
\end{aligned}
$$

where $\kappa=1$ for unit intensity. The response PDF $P(X, t)$ can be obtained by numerically applying the iterations in Eq. (10) (Naess and Johnsen 1991; Lin and Yim 1995).

\section{Experimental Observations}

One of the primary goals of the experimental investigation reported by Yim et al. (1993) was to examine the effects of various degrees of randomness in the exciting waves on the highly nonlinear model responses. For demonstration purposes, representative sample tests with various wave conditions, including nearly periodic ("deterministic" waves with tank noise), noisy periodic (monochromatic waves with designed perturbations), and narrowband random waves are shown for reference purposes.

A sample of a nearly periodic wave profile and its corresponding response time history (test D2) are shown in Figs. 2(a and b), respectively. The test was originally designed to be deterministic, however, because of the presence of uncontrollable weak tank noise, variations in wave amplitude are noted [Fig. 2(a)]. The variations in the wave profile cause a transition between two distinct response modes, from harmonic response to subharmonic,

a)

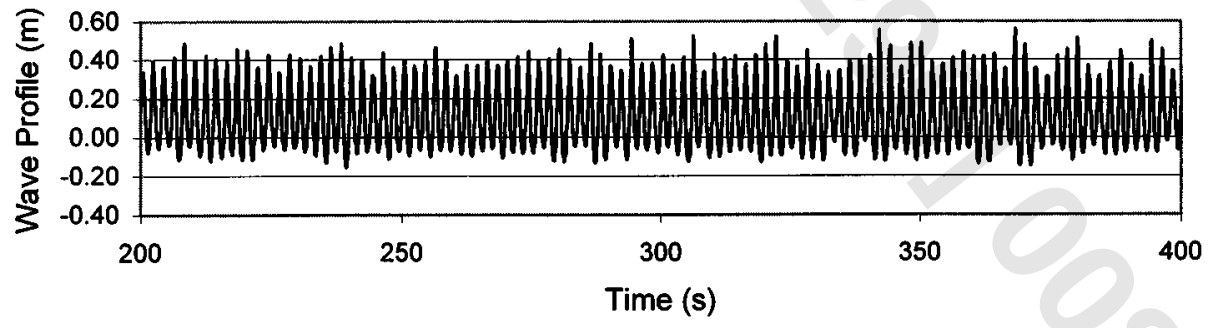

b)

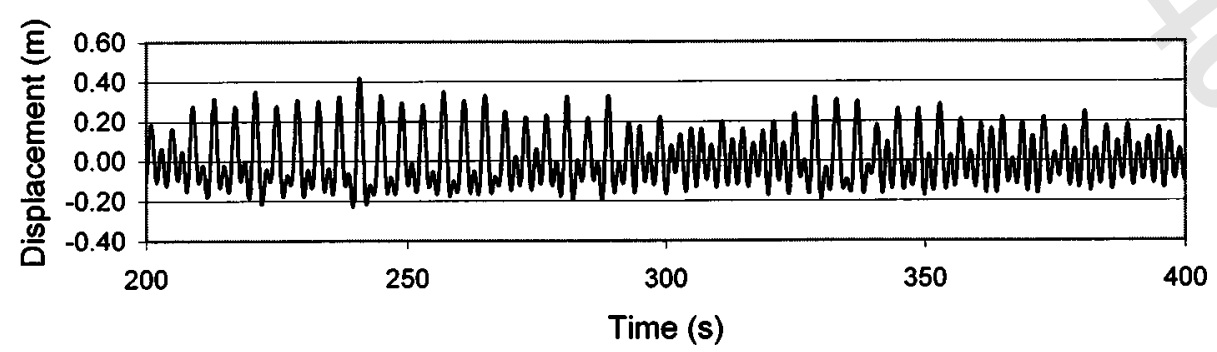

Fig. 3. Oscillations between coexisting harmonic and subharmonic response attractors (test D11): (a) wave profile and (b) sphere displacement 
a)

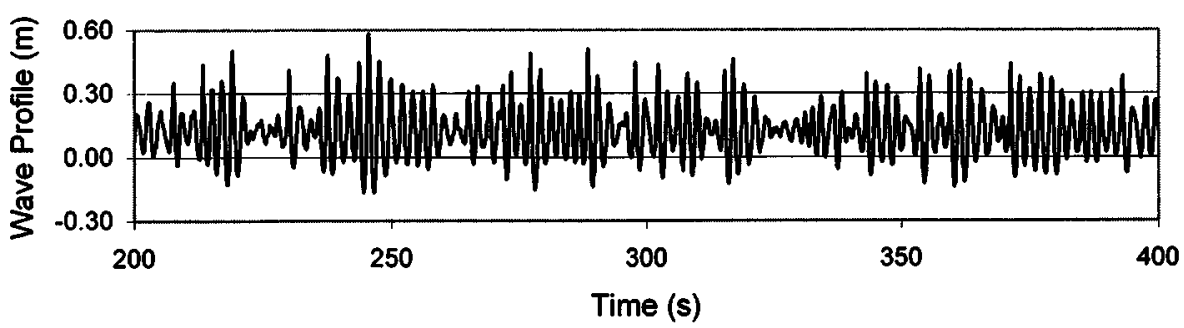

b)

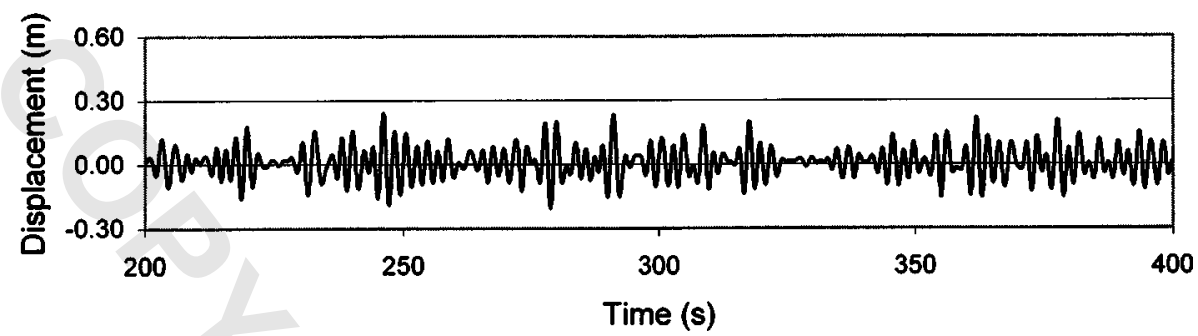

Fig. 4. Response subjected to narrow-band random excitation (test D16): (a) wave profile and (b) sphere displacement
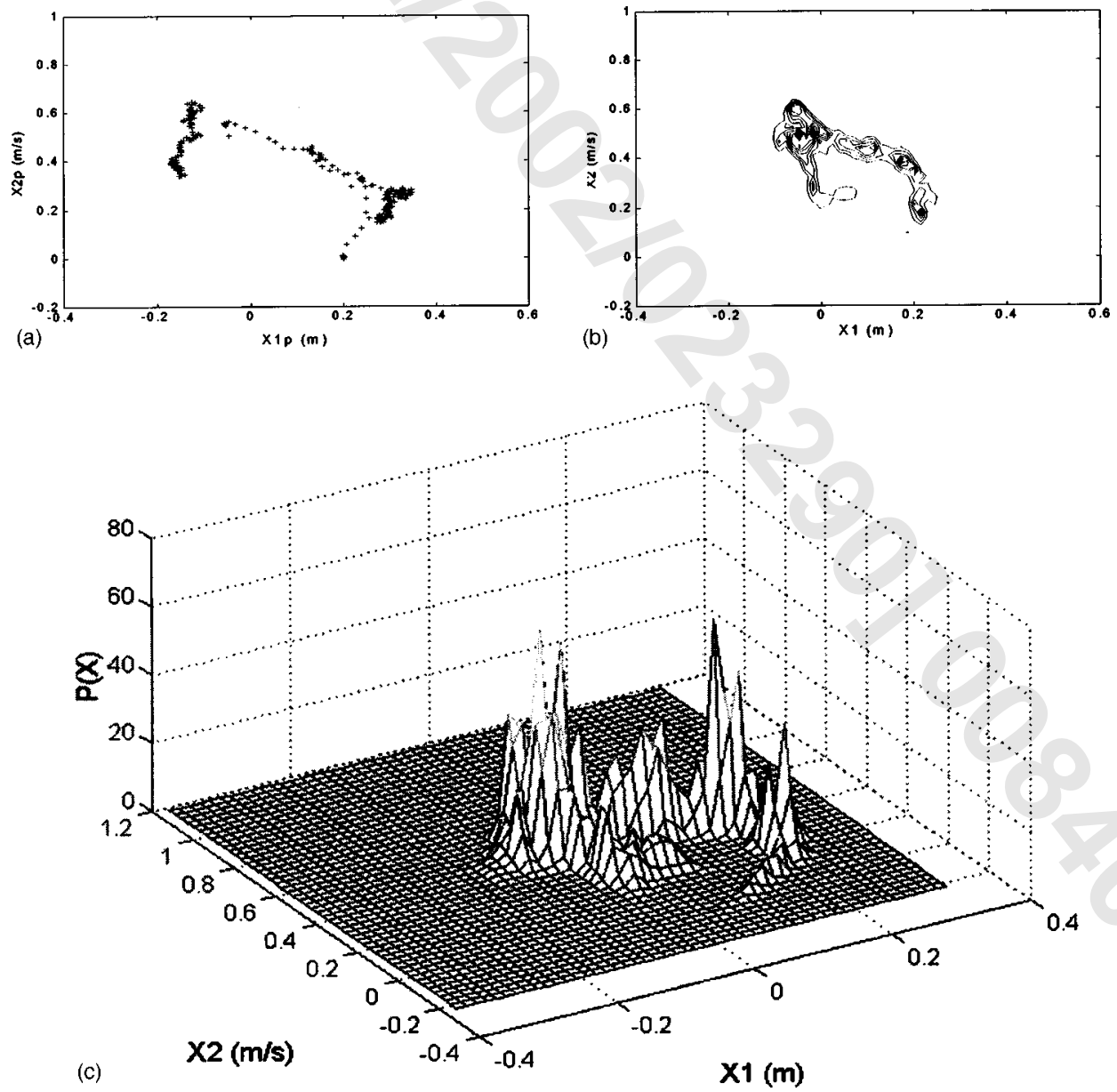

Fig. 5. Coexisting harmonic and subharmonic responses: (a) experimental result in Poincaré map (test D2); (b) simulations (contour map of PDF); and (c) 3D PDF 


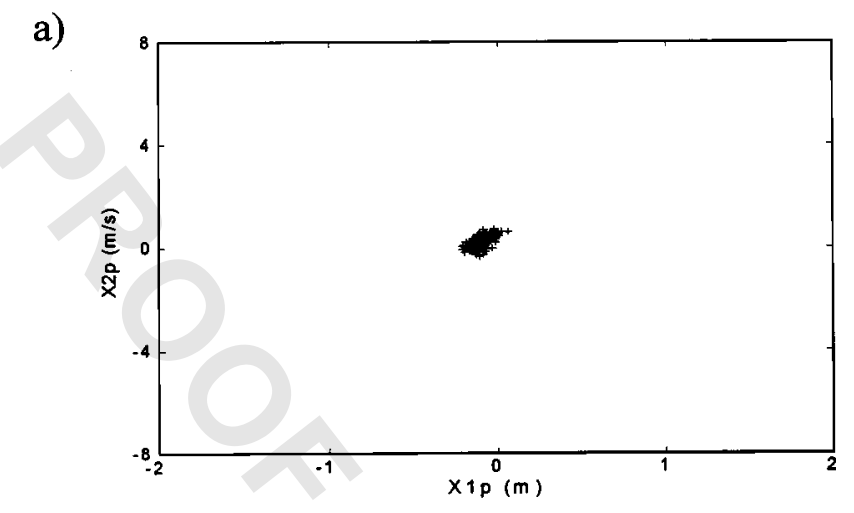

b)

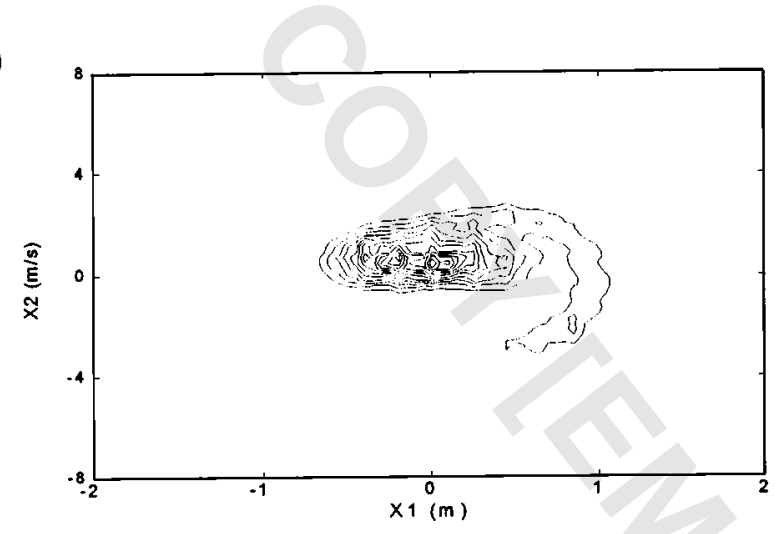

Fig. 6. Coexisting small-amplitude harmonic, subharmonic, and large-amplitude harmonic responses in PDF: (a) experimental results in Poincaré map (test D11); (b) simulations (contour map)

observed at around 120th $\mathrm{s}$ in the time history [Fig. 2(b)].

A sample of a wave profile with weak additive perturbations and its corresponding response time history (test D11) are shown in Figs. 3(a and b), respectively. The test was designed with weak additive random perturbations in the waves to examine their effects on the structural response. As expected, there are more notable variations in wave amplitude [Fig. 3(a)]. Based on the time domain analysis and predictions by Yim and Lin (2002), two response attractors, i.e., harmonic and subharmonic, are observed to coexist. Because of the presence of random perturbations in waves, experimental responses exhibit interactions of the two coexisting response characteristics in the time history [Fig. 3(b)]. In addition, occurrences of large amplitude responses beyond the limitations of the experimental configuration were also observed and noted. No time histories of those large-amplitude responses were recorded because their amplitudes were out of the reading ranges of experimental apparatuses.

A sample of a narrow-band random wave profile and its corresponding response time history (test D17) are shown in Figs. 4(a and b), respectively. The wave excitation of the test was designed to be random and narrow-banded [Fig. 4(a)]. The response also exhibits the inherited, random, and narrow-banded nature as shown in Fig. 4(b).

Note that no apparent, chaos-like, SDOF experimental results had been identified. A recent simulation study on the SDOF, IFF model has been conducted to identify all possible nonlinear responses (Yim and Lin 2002). Numerical results indicated that the diminishing of chaotic response may be caused by a relatively high structural damping, due to the presence of the rod (cf. Fig. 1). a)

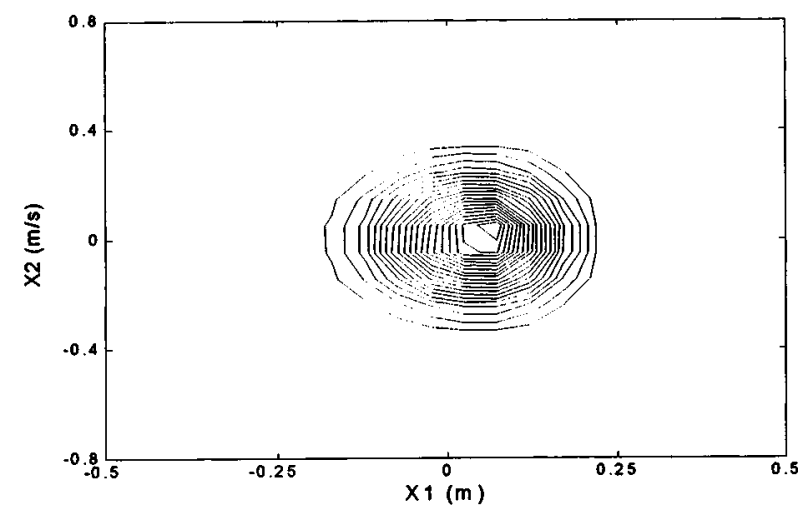

b)

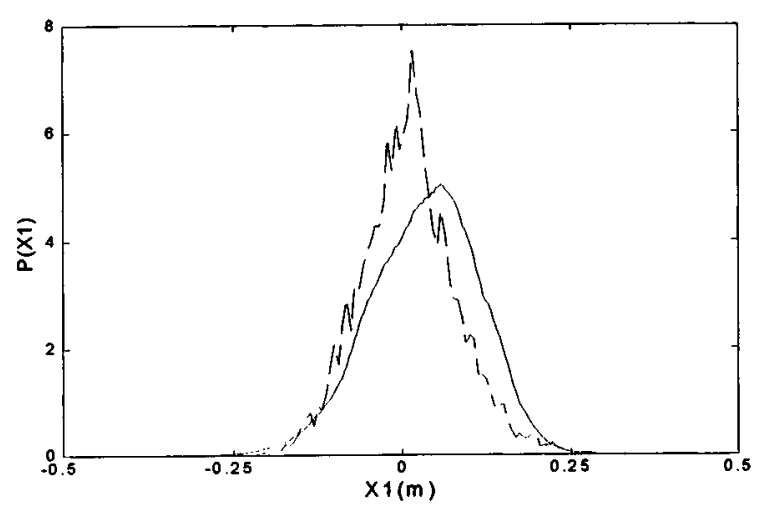

Fig. 7. Response PDF to narrow-band random waves: (a) simulations (contour map) and (b) comparisons in marginal PDF; experimental result (test D17, dashed line) and simulation (solid line)

These tests are studied, compared, and interpreted in the following section from a probabilistic perspective via their associated response PDFs.

\section{Comparisons and Analyses}

Experimental results and FPE PDF predictions are compared either on a Poincare section or in margin PDFs for demonstration purposes. The experimental results under nearly periodic or noisy periodic waves are sampled by a Poincaré map and compared with the FPE predictions (joint PDFs) on the Poincare section. A Poincaré map is formed by sampling and collecting response data at every forcing period of the dominant periodic excitation component. Concentrations of the Poincare points indicate the locations of possible (co-) existing response attractors. Probabilistic characteristics of the responses subjected to narrow-band random waves are represented by a histogram and compared with the corresponding marginal PDFs. Asymptotic long-term behaviors of the experimental results as demonstrated in the previous section are also examined and inferred.

\section{Nearly Periodic Waves (Test D2)}

The Poincare map of the response subjected to nearly periodic waves (test D2) is shown in Fig. 5(a). The concentration of Poincaré points indicates two coexisting response attractors, i.e., harmonic and subharmonic. The harmonic attractor is located near 
a)

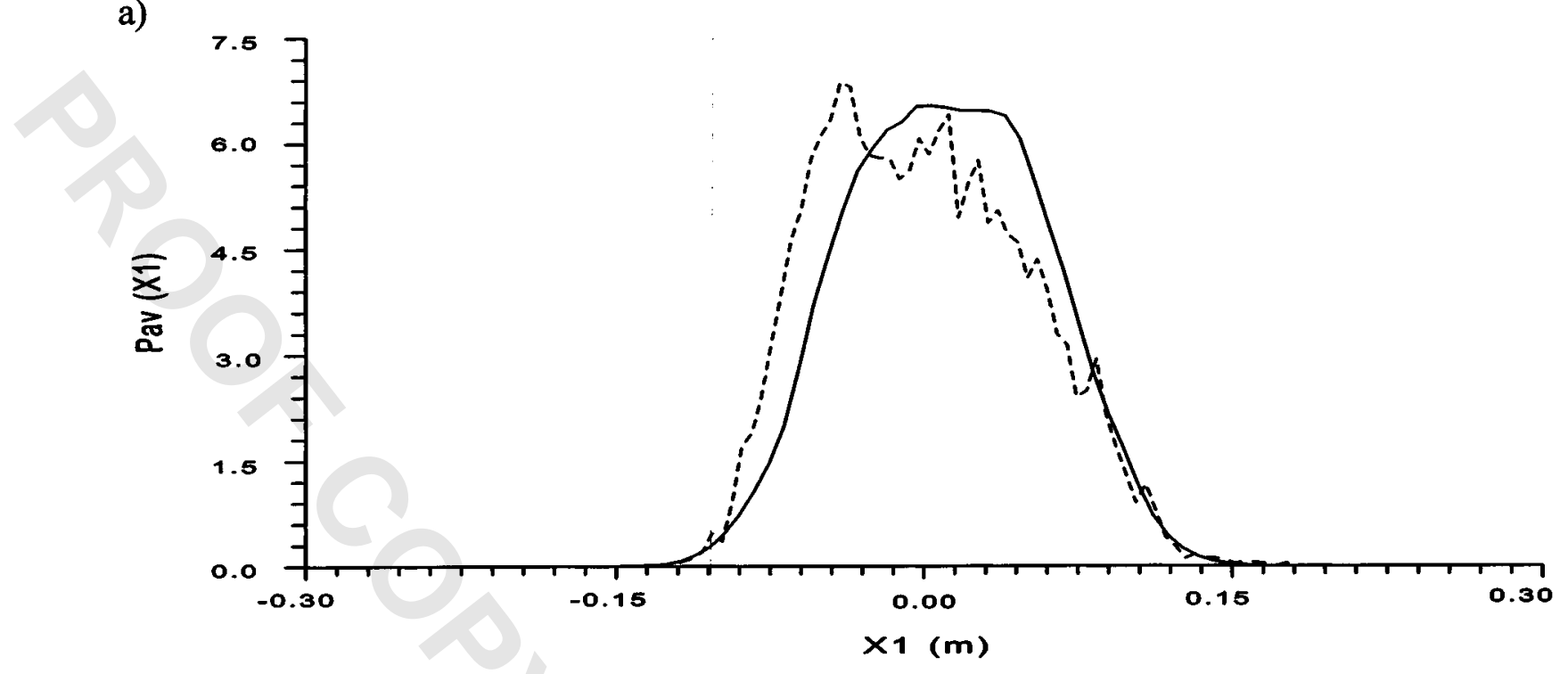

b)

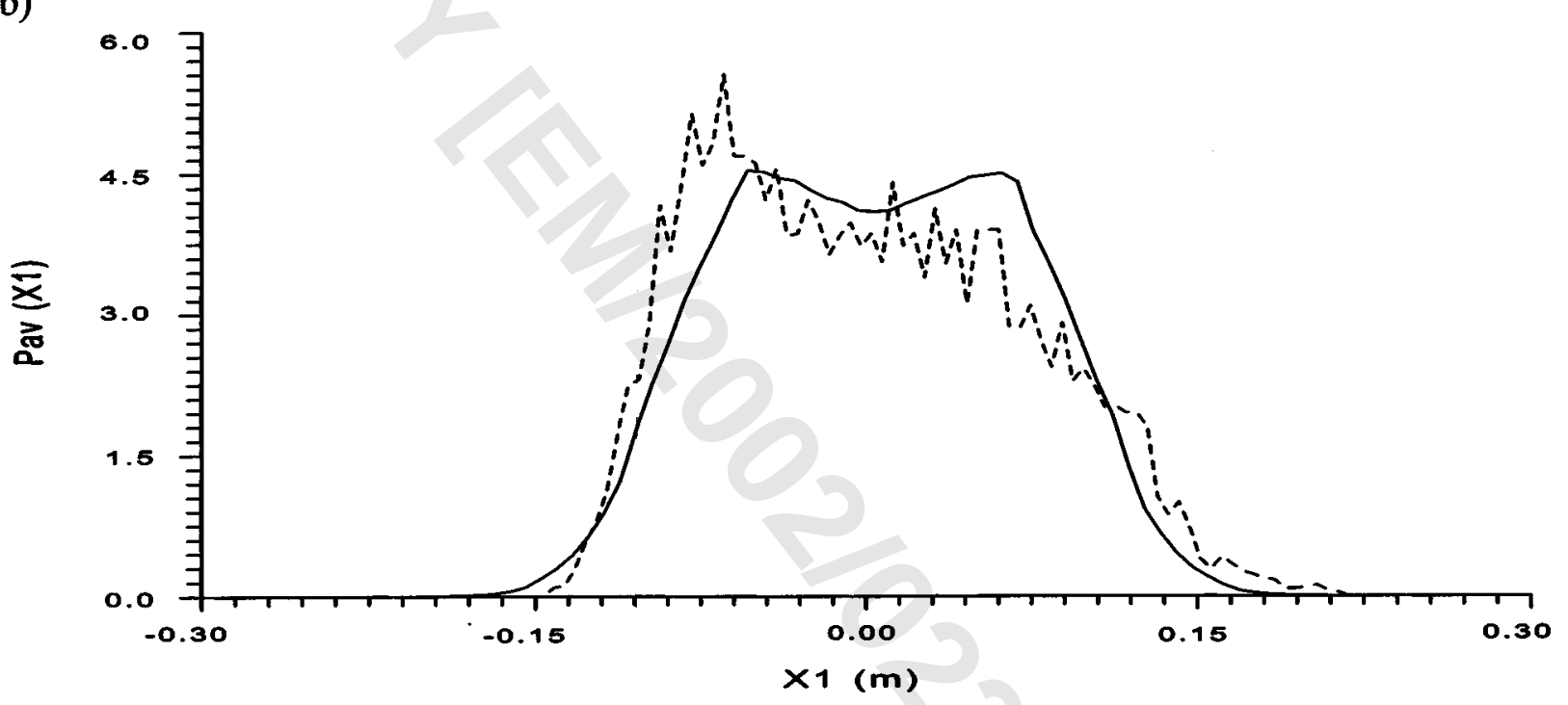

Fig. 8. Time-averaged probability density functions: (a) test D7 $\left(\delta_{\zeta}=0.03\right)$ and (b) test D10 $\left(\delta_{\zeta}=0.05\right)$; dashed lines: experimental results, and solid lines: analytical predictions

$(0.1,0.45)$, and the subharmonic attractor is shown by the two concentrations with one near $(-0.15,0.60)$ and the other near $(0.30,0.15)$. The corresponding joint response PDF on the Poincaré section by the contour map is shown in Fig. 5(b). The PDF can fully capture the coexisting attractors and their relative strengths. The relative strengths are better demonstrated by the magnitude of PDF in a three-dimensional presentation as shown in Fig. 5(c). Comparatively higher magnitude is shown in the subharmonic domain [two peaks near $(-0.15,0.60)$ and $(0.30,0.15)]$, indicating the subharmonic response as the stronger attractor.

As experimentally observed, because of the presence of weak tank noise bridging the coexisting attractors, the response trajectory is directed to and stays in the stronger (subharmonic) attractor as shown in Fig. 2(b).

\section{Periodic Waves with Weak Perturbations (Test D11)}

The Poincare map of the response subjected to periodic wave with additive weak perturbations (test D11) is shown in Fig. 6(a).
The clustering of the Poincare points appears to indicate a single existing response attractor. However, the corresponding response PDF on the Poincare section by the contour map [Fig. 6(b)] indicates the coexistence of two attractors [as shown in the measured response time history of the experimental result in Fig. 3(b)] in the area where the experimental Poincaré points are located. The PDF can fully capture the coexisting harmonic [near $(0,0)]$ and subharmonic response attractors [two peaks at $(-0.3,0)$ and $(0.3,0)$, respectively] and their relative strengths. It is also noted that in addition to the experimentally observed two attractors, namely small-amplitude harmonic and subharmonic, time-domain simulations indicated the existence of another large-amplitude harmonic response. The large-amplitude harmonic attractor near $(1,-3.5)$ is captured in the corresponding PDF, which also provides information of its relative strength [Fig. 6(b)]. All three coexisting attractors are well bridged due to the presence of a moderate level of random perturbations in the waves.

Comparatively, the subharmonic response is the strongest attractor, and the large-amplitude harmonic the weakest. It is antici- 


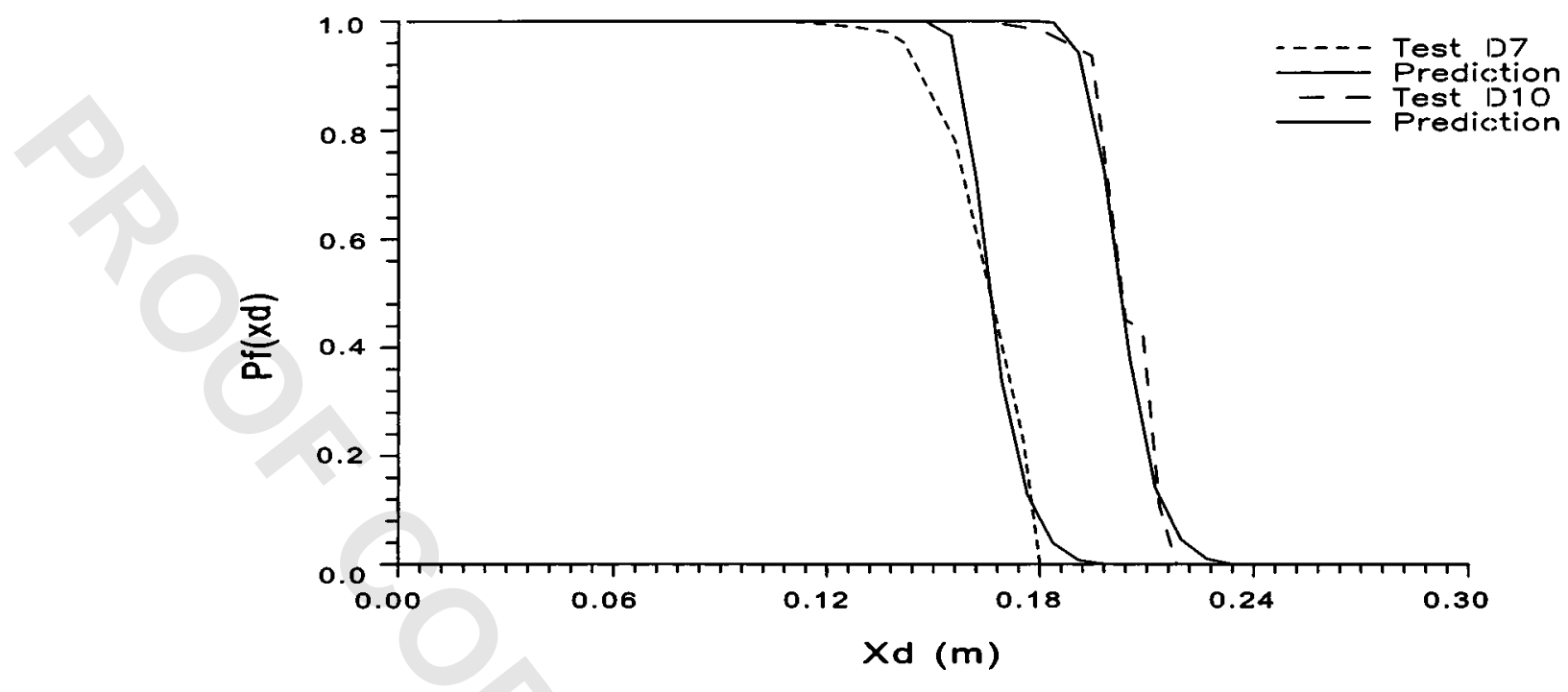

Fig. 9. Distribution of large excursions; dashed lines: experimental results (test D7 with $\delta_{\zeta}=0.03$, and test D10 with $\delta_{\zeta}=0.05$ ), and solid lines: corresponding analytical predictions

pated that with the quiescent initial conditions, the response trajectory is much less likely to reach the large-amplitude domain. The prediction is in agreement with the experimental observation that the response trajectory mostly oscillates between the two stronger attractors with very low tendency reaching the largeamplitude as shown in Fig. 3(b). Nonetheless, in a deterministic continuous search test intended to examine the nonlinear phenomenon through the full wave frequency range considered, tendency of reaching a large-amplitude response is observed when the wave frequency is near $0.27 \mathrm{~Hz}$. In that case, large initial displacement and velocity are virtually incorporated. The prediction of the existence of a large-amplitude harmonic response confirms the experimental observation.

\section{Narrow-Band Random Waves (Test D17)}

The contour map of the response PDF subjected to narrow-band random waves (test D17) sampled on the Poincare section is shown in Fig. 7(a). The response PDF captures the distribution of the attracting domain. A comparison of the experimental result and prediction is carried out in the associated marginal PDF as shown in Fig. 7(b). Reasonably good agreement between the experimental and FPE results is observed. Note that the numerical PDF overestimates slightly at the tails, which leads to a conservative estimation on the distribution of large excursions as demonstrated in a later section.

\section{System Performance}

The probability distribution of large excursions in the response process is important information for reliability-based engineering design. To experimentally estimate the probability distribution of the large excursions, a large number of samples is needed. However, in reality, the sample size is generally limited due to costs, schedules, and operations (for the specific experiment studied here, a significant amount of time was devoted to deterministic cases and "trial runs" for random cases for which data were not collected). A combined analysis and prediction procedure of computing the invariant measure of the experimental results and employing an asymptotic approximation of the response up-crossing frequency over a specified amplitude is used here to estimate the distribution. Experimental results are compared with the numerical predictions.

\section{Invariant Measure}

Time-averaged PDFs have been identified to be an invariant measure of the nonlinear stochastic responses (Jung and Hänggi 1990). The invariant measure $P_{\mathrm{av}}(X)$ is obtained by taking timeaverage over a suitable long duration to suppress the periodicity caused by the dominant periodic excitation component.

$$
P_{\mathrm{av}}(X)=\frac{1}{T} \int_{0}^{T_{n}} P(X, t) \mathrm{d} t
$$

and the invariant measure on $x_{1}$ (i.e., marginal PDF) is given by

$$
P_{\mathrm{av}}\left(x_{1}\right)=\int_{-\infty}^{\infty} P_{\mathrm{av}}\left(x_{1}, x_{2}\right) \mathrm{d} x_{2}
$$

Fig. 8 shows a comparison of the invariant measures on $x_{1}$ of experimental results (tests D7 and D10) and the corresponding analytical predications (Fokker-Planck formulation). Tests D7 and D10 were subjected to an "identical" periodic excitation component with two various perturbation intensities, $\sigma_{\xi} \approx 0.03$ and 0.05 , respectively. Good agreements between the experimental results and analytical predications are observed in both Figs. $8(\mathrm{a}$ and $\mathrm{b})$. It also shows that the invariant measure is smoothed and widespread when the noise intensity is large. The invariant measure is then utilized in the following section to estimate the asymptotic behavior of the response up-crossing frequency at a specified amplitude level, and the probability of exceedance can be evaluated.

\section{Distribution of Large Excursions}

Using the invariant measure and employing Rice's formula, the mean up-crossing frequency of the response $\mu_{x_{1}}^{+}\left(x_{d}\right)$ can be evaluated as follows:

$$
\mu_{x_{1}}^{+}\left(x_{d}\right)=\int_{0}^{\infty} x_{2} P_{\mathrm{av}}\left(x_{d}, x_{2}\right) \mathrm{d} x_{2}
$$


By adopting the assumption of statistically independent largeamplitude up-crossings, which leads to Poisson-distribution crossing events (Naess and Johnsen 1993), the asymptotic approximation of the probability $P_{F}$ that $x_{1}$ exceeds a specified high level $x_{d}$ during time $T$ is given by

$$
P_{F}\left(x_{d}, T\right)=1-\exp \left[-\mu_{x_{1}}^{+}\left(x_{d}\right) T\right]
$$

Fig. 9 shows both the experimental and analytical estimations of the distribution of large response displacements subjected to an "identical" periodic wave excitation component with random noise of various intensities over the duration of $300 \mathrm{~s}$ (tests D7 and D10). Good agreements between the experimental results and the corresponding analytical predictions are observed in both tests (Fig. 9). It is also shown that the probability distribution curve is elevated when the noise intensity is increased. Thus probability of large excursions occurring in the structural response increases with noise intensity, as expected.

\section{Concluding Remarks}

This study presents probability-based analyses and comparisons of experimental responses of a moored, submerged structural system with hydrodynamic and geometrical nonlinearities subjected to various types of random wave excitations. Based on the results presented, the following concluding remarks are drawn.

Good agreement is shown between experimental and numerical results in the response probability density functions subjected to various types of random wave excitations considered, including nearly periodic, noisy periodic, and narrow-band random waves.

Response probability density functions, depicting all of the potential coexisting attractors and indicating their relative strengths, provide the global information about the experimental response behavior.

Numerical results, confirmed by experimental data, predict that when the noise intensity is low, the response trajectory is led to and stays in the stronger coexisting attractor. When the noise intensity is moderate, the coexisting attractors are well bridged and the response trajectory oscillates between the attractors in accordance with their relative strengths.

Potential existence and the strength of a large-amplitude harmonic response are identified in the response density function. Its comparatively low strength indicates that with quiescent initial conditions the response trajectory has a low probability of visiting this large amplitude attractor. The prediction confirms the experimental result of the sphere's reaching a large amplitude motion.

Using a time-averaged probability density function of the model responses as an invariant measure, distributions of large excursions are estimated. Good agreements between experimental results and the corresponding analytical predictions are demonstrated. The probability of the system experiencing large displacements is found to increase with increasing noise intensity.

\section{Acknowledgment}

The writers gratefully acknowledge the financial support from the United States Office of Naval Research (Grant No. N00014-92-J1221).

\section{References}

Gottlieb, O., and Yim, S. C. S. (1992). "Nonlinear oscillations, bifurcations and chaos in a multipoint mooring system with a geometric nonlinearity." Appl. Ocean. Res., 14, 241-257.

Gottlieb, O., and Yim, S. C. S. (1993). "Drag-induced instability and chaos in mooring systems." Ocean Eng., 29, 569-599.

Gottlieb, O., Yim, S. C. S., and Lin, H. (1997). "Analysis of bifurcation superstructure of nonlinear ocean system." J. Eng. Mech., 123(11), $1180-1187$.

Jung, P., and Hänggi, P. (1990). "Invariant measure of a driven nonlinear oscillator with external noise." Phys. Rev. Lett., 65, 3365-3368.

Lin, H., and Yim, S. C. S. (1995). "Chaotic roll motion and capsizing of ships under periodic excitation with random noise." Appl. Ocen. Res. 17, 185-204.

Lin, H., and Yim, S. C. S. (1997). "Noisy nonlinear motions of moored system. I: Analysis and simulations." J. Waterw., Port, Coastal, Ocean Eng., 123(5), 287-295.

Lin, H., and Yim, S. C. S. (1998). "Experimental calibration of bifurcation superstructure of nonlinear system." J. Eng. Mech., 124(4), 471475.

Lin, H., Yim, S. C. S., and Gottlieb, O. (1998). "Experimental investigation in bifurcations of an ocean system." Ocean Eng., 254, 323-343.

Naess, A., and Johnsen, J. M. (1991). "Statistics of nonlinear dynamic systems by the path integrations." Nonlinear Stochastic Mechanics, IUTAM Symposium, Turin, 401-409.

Naess, A., and Johnsen, J. M. (1993). "Response statistics of nonlinear, compliant offshore structures by the path integral solution method." Probab. Eng. Mech., 8, 91-106.

Narayanan, S., and Yim, S. C. S. (2000). "Nonlinear model evaluation via system identification of a moored structural system." Proc., 10th ISOPE Conf., Seattle, II, 403-409.

Soliman, M., and Thompson, J. M. T. (1991). "Transition and steady state analysis of capsize phenomena." Appl. Ocean. Res., 13, 82-92.

Thompson, J. M. T. (1989). "Chaotic phenomena triggering the escape from a potential well." Proc. R. Soc. London, Ser. A, 421, 195-225.

Virgin, L. N., and Bishop, S. R. (1988). "Complex dynamics and chaotic responses in the time domain simulations of a floating structure." Ocean Eng., 15, 7-90.

Virgin, L. N., and Erickson, B. K. (1994). "A new approach to the overturning stability of floating structures." Ocean Eng., 21, 67-80.

Yim, S. C. S., and Lin, H. (2000). "Noisy nonlinear motions of moored system. II: Experimental study." J. Waterw., Port, Coastal, Ocean Eng., 126(3), 113-120.

Yim, S. C. S., and Lin, H. (2002). "An independent flow field model for SDOF nonlinear structural system. I: Identification and comparisons." J. OMAE (in press).

Yim, S. C. S., Myrum, M. A., Gottlieb, O., Lin, H., and Shih, I.-M. (1993). "Summary and preliminary analysis of nonlinear oscillations in a submerged mooring system experiment." Report No. OE-93-03, Ocean Engineering Program, Oregon State Univ. 